\title{
Sarcome Uterin about a Case and Literature Review
}

\author{
Melhaoui Houda*, Maggouh Karima, Rachid Bezad, Alami MH
}

Motherhood of orange Trees, CNSR flap, Mororocco

DOI: $10.36348 /$ sijog.2020.v03i02.007 | Received: 08.02.2020 | Accepted: 15.02.2020 | Published: 19.02 .2020

*Corresponding author: Melhaoui Houda

\section{Abstract}

Uterine sarcomas are rare tumours characterized by clinical and histopathological diversity and poor prognosis. We analyzed diagnostic, prognostic and therapeutic difficulties encountered with these tumors by insisting on the importance of early diagnosis.

Keywords: Uterine sarcoma, Extemporaneous examination, Surgery, Prognostic factors.

Copyright @ 2020: This is an open-access article distributed under the terms of the Creative Commons Attribution license which permits unrestricted use, distribution, and reproduction in any medium for non-commercial use (NonCommercial, or CC-BY-NC) provided the original author and source are credited.

\section{INTRODUCTION}

Uterine sarcomas are rare tumors that represent 3 to $5 \%$ of malignant tumors of the uterus [13]. They are characterized by a diversity histopathological and clinical important. We distinguishes 3 histological types: leiomyosarcomas, mixed Mullerian sarcomas and sarcomas of endometrial stroma (also called sarcomas of the cytogenous chorion) [3].

Their diagnosis is not very often made histopathological analysis of the hysterectomy or myomectomy [4]. The main factors prognostics are stage, grade and age [4-7]. Their treatment is primarily surgical. Radiotherapy Postoperative increases local control. The Chemotherapy is reserved for metastatic stages. The prognosis is generally poor, with a local recurrence between 50 and $70 \%$ and an evolution metastatic (willingly pulmonary) in $70 \%$ of cases $[3,8]$.

The purpose of our work is to clarify the aspects histological, clinical, therapeutic and prognostic uterine sarcomas

\section{PATIENT AND OBSERVATION}

This is a 40-year-old patient with no history pathological disease, who had presented 3 years of pelvic pain associated with menometrorrhagia. The clinical examination finds a uterus of 12sa. Ultrasonography reported an intracavitary image with a dual fluid and tissue component with a heterogeneous appearance with scattered Doppler vascularization and a $4 \mathrm{~mm}$ safety wall.

Given the age of the patient and the presence of a single fibroma suggestive of uterine sarcoma, the abdominipelvic MRI was asked to demonstrate the presence of a type 0 intracavitary myoma according to FIGO and absence of abdominal evolutionary lesion.

The surgical procedure performed was a myomectomy, at the analysis anatomopathological, the appearance of a grade 3 endometrioid adenocarcinoma infiltrating the myometrium.

The patient subsequently benefited from a type a colpohysterctomy with bilateral pelvic lymph node dissection with an anapathic endometrioid grade 3 adenocarcinoma largely infiltrating more than $50^{\circ} /{ }^{\circ}$ of the myometrium and arriving focal $0.2 \mathrm{~cm}$ from the uterine serosa and absence of vascular embolism, i.e. T1b N0

\section{DISCUSSION}

Uterine sarcomas are an entity pathological relatively rare. The incidence appears increased in recent years. They are no longer considered very rare tumors. This is due on the one hand to a better knowledge of the different pathological aspects of uterine sarcomas especially with the development of immunohistochemistry and on the other hand to the exposure more and more to different predisposing 
factors like irradiation pelvic and use of Tamoxifen in cancer of breast [1].

The epidemiology of these tumors are disparate. The incidence was estimated between 4 and $9 \%$ of tumors malignant uterines in the most recent studies [2- 4]. Uterine sarcomas derive from the elements Mesenchymatous constituent of the uterine body: the muscle smooth and endometrial stroma. They can associate uterine epithelial tumors giving rise to mixed tumors. Other tumor types (angiosarcoma, rhabdomyosarcoma ...) are more rare and not specific to the uterus. WHO classification [5, 6] distinguishes four groups: leiomyosarcomas, mixed Mullerian tumors including homologous tumors (carcinosarcomas) and heterologous tumors (mixed tumors mesodermal), endometrial stromal sarcomas belonging to tumors of the cytogenous chorion and finally unclassifiable tumors. The incidence of each type histological is variously estimated by the authors. In general, carcinosarcomas and leiomyosarcomas constitute the uterine sarcomas more met They represent about $80 \%$ of uterine sarcomas. The endometrial stromal sarcoma is the third type tumor and account for $15 \%$ of the total. The unclassifiable sarcomas, usually of high grade malignancy, constitute the rest [1]. Uterine sarcomas affect women from the pubertal period to postmenopause. The average age of onset varies in the literature from 50 to 65 years old with extremes ranging from 15 at 85 years old. It has been observed a greater frequency of carcinosarcomas and a higher histological grade in older patients. On the contrary, patients younger people had more frequent tumors of Low histological grade and leiomyosarcomas [2, 5, 7, 8]. Clinical manifestations are not specific. The symptomatology can imitate especially that of uterine fibroids. Thus, the menometrorrhagia are encountered in 45 to $86 \%$ of cases and pain pelvic in 20 to $50 \%$ of cases [4]. Elsewhere, uterine sarcomas may be in the form of a necrotic tumor, delivered by the cervix [3].

ER diagnosis is often difficult; he must be evokes before any rapid increase in volume of a myoma, a rapidly recurrent myoma or a necrotic tumor delivered by the cervix [4].

The imagery is not very specific. The ultrasound aspect does not differ from that of a fibroid. However, the reshaped fibroma aspect is most often meet. Computed tomography is not very specific and does not distinguish leiomyosarcoma from fibroma in necrobiosis [8]. Magnetic resonance imaging allows in certain situations to guide the diagnosis, especially in cases of atypical uterine mass to classical imagery. It also allows differentiate leiomyoma from leiomyosarcoma [9].

Therapeutically, surgery constitutes the first stage of management of uterine sarcomas. The reference surgical procedure is hysterectomy with bilateral adnexectomy in case of tumor a priori limited to the uterine body. In the carcinosarcomas, omentectomy and pelvic lymphadenectomy should be associated systematically given the frequency of ectopic localizations especially ganglionic and peritoneal [10, 12]. In other types histological, omentectomy and lymphadenectomy should be performed when there are injuries Suspicious epiploon and / or adenomegaly discovered during surgical exploration [13,14].

The chosen approach must allow the removal of the uterus in one piece to reduce the risk of peritoneal dissemination or dissemination early postoperative vaginal discharge. Moreover, the way first chosen must allow to achieve the others excisional gestures or biopsies needed. The laparotomy seems to be the most appropriate approachwhen the diagnosis of uterine sarcoma is known or suspected before the procedure [15]. In tumors of more advanced stage (involvement of the rectum or bladder, peritoneal involvement, distant metastasis), surgery is discussed; nevertheless, the hysterectomy with Bilateral adnexectomy seems appropriate when it is technically feasible. If surgery remains the primary treatment for localized sarcomas, the adjuvant treatment remains discussed. Most of authors agree that irradiation adjuvant brings a benefit in terms of control local. Even if the benefit on survival is not Certainly, a decrease in the number of recurrences pelvic, often with pain, may justify the prescription of adjuvant radiotherapy. This benefit is often expected for tumors of high histological grade [16-17]; About the chemotherapy, its interest remains uncertain, although that it is probably recommended in specific situations (young patients, high FIGO stage, histological grade III). It should not be too delay irradiation $[17,18]$. The prognosis of Uterine sarcomas remains until our dark days. After surgery alone, 5-year survival was estimated at 6 to 42 years \% $[5,18,20]$ according to the different series, in any case lower than that of patients treated for carcinoma of the endometrium [19].

Prognostic factors have been studied extensively in the litterature. The main factors reported were the hormonal status, the histological type, the grade histological, the clinical stage and the presence of a tumor residue after surgery. Carcinosarcomas have the worst prognosis followed by the leiomyosarcomas and stroma sarcomas endometrial [21]. However, leiomyosarcomas recurred more than other sarcomas.

\section{CONCLUSION}

SUs are rare tumors. Their diagnosis is often late. Overall prognosis remains gloomy despite therapeutic advances. Surgery is gold standard of these tumors. Prognostic factors main are the histological type, the stage extension, mitotic activity for leimyosarcomas and the presence of a residue after surgery [21, 22]. Waiting final results prospective studies outstanding, adjuvant treatment reference 
Melhaoui Houda et al; Sch Int J Obstet Gynec, Feb. 2020; 3(2): 48-50

remains on external radiotherapy pelvic, which increases local control. The place of the brachytherapy of the vaginal fund remains to be defined. The chemotherapy is currently reserved for stadiums metastatic. The place of targeted therapy and / or hormone therapy remains to be defined

\section{REFERENCES}

1. Reed, N.S. (2002). Uterine sarcomas: the biggest challenge Clin Oncol, 14: 50-53.

2. Brooks, S.E., Zhan, M., Cote, T., Baquet, C.R. (2004). Surveillance, epidemiology, and end results analysis of 2677 cases of uterine sarcoma 19891999. Gynecol Oncol, 93: 204-8.

3. Nordal, R., Thoresen, S. (1997). Uterine sarcomas in Norway 1956- 1962: incidence, survival and mortality. Eur J Cancer, 33: 907-11.

4. Harlow, B., Weiss, N., Lofton, S. (1986). The epidemiology of sarcomas of the uterus. JNCI, 76: 399-402.

5. Michel, G., Pfeiffer, F., Duvillard, P., Prade, M., Castaigne, D., Zarca, D. (1989). Sarcomes utérins. Étude clinique à propos de 50 cas opérés à l'Institut Gustave Roussy. J Gynecol Obstet Biol Reprod, 18: 1024-30.

6. World Health Organisation.(1975). Histological Typing of female genital tract tumours. Poulsen HE, Taylor CW, Eds, WHO, Genève, 13.

7. Deniaud-Alexandre, E., Chauveine, L., Rochefordiere, A., Sastre, X., Clough, K.B. (2001). Intérêt des traitements adjuvants dans les sarcomes utérins : expérience de l'institut Curie. cancer Radiother, 6: 743-9.

8. Livi, L., Andreopoulou, E., Shah, N., Paiar, F., Blake, P., Judson, I., \& Harmer, C. (2004). Treatment of uterine sarcoma at the Royal Marsden Hospital from 1974 to 1998. Clinical Oncology, 16(4), 261-268.

9. Corson, S. L., \& Brooks, P. G. (1991). Resectoscopic myomectomy. Fertility and Sterility, 55(6), 1041-1044.

10. Wm, C. (1972). Williamson EO, Gray LA: Leiomyosarcoma of the uterus. Cancer, 9, 15321517.

11. Razafintsalama, T., Leveque, J., Le Gall, F., Paumier, V., \& Pangui, E. (1997). Le léiomyosarcome utérin: A propos de 9 observations, revue de la littérature. Journal de gynécologie obstétrique et biologie de la reproduction, 26(3), 256-262.
12. Schwartz, L. B., Diamond, M. P., \& Schwartz, P. E. (1993). Leiomyosarcomas: clinical presentation. American journal of obstetrics and gynecology, 168(1), 180-183.

13. Schwartz, L. B., Diamond, M. P., \& Schwartz, P. E. (1993). Leiomyosarcomas: clinical presentation. American journal of obstetrics and gynecology, 168(1), 180-183.

14. Kurjak, A., Kupesic, S., Shalan, H., Jukic, S., Kosuta, D., \& Ilijas, M. (1995). Uterine sarcoma: a report of 10 cases studied by transvaginal color and pulsed Doppler sonography. Gynecologic oncology, 59(3), 342-346.

15. Hata, K., Hata, T., Makihara, K., Aoki, S., Takamiya, O., Kitao, M., ... \& Nagaoka, S. (1990). Sonographic findings of uterine leiomyosarcoma. Gynecologic and obstetric investigation, 30(4), 242-245.

16. Morice, P., Rodrigues, A., Pautier, P., Rey, A., Camatte, S., Atallah, D., ... \& Castaigne, D. (2003). Chirurgie des sarcomes utérins: revue de la littérature et recommandations sur la prise en charge chirurgicale. Gynécologie obstétrique \& fertilité, 31(2), 147-150.

17. Janus, C., White, M., Dottino, P., Brodman, M., \& Goodman, H. (1989). Uterine leiomyosarcomamagnetic resonance imaging. Gynecologic oncology, 32(1), 79-81.

18. Takemori, M., Nishimura, R., \& Sugimura, K. (1992). Magnetic resonance imaging of uterine leiomyosarcoma. Archives of gynecology and obstetrics, 251(4), 215-218.

19. Rha, S. E., Byun, J. Y., Jung, S. E., Lee, S. L., Cho, S. M., Hwang, S. S., ... \& Lee, J. M. (2003). CT and MRI of uterine sarcomas and their mimickers. American Journal of Roentgenology, 181(5), 1369-1374.

20. Leibsohn, S., d'Ablaing, G., Mishell, D. R., \& Schlaerth, J. B. (1990). Leiomyosarcoma in a series of hysterectomies performed for presumed uterine leiomyomas. American Journal of Obstetrics \& Gynecology, 162(4), 968-976.

21. Oda, K., Okada, S., Nei, T., Shirai, T., Takahashi, M., Sano, Y., \& Shiromizu, K. (2004). Cytodiagnostic problems in uterine sarcoma. Acta cytologica, 48(2), 181-186.

22. Kawamura, N., Ichimura, T., Ito, F., Shibata, S., Takahashi, K., Tsujimura, A., ... \& Ogita, S. (2002). Transcervical needle biopsy for the differential diagnosis between uterine sarcoma and leiomyoma. Cancer, 94(6), 1713-1720. 